\title{
Erratum to: A Tailored Finite Point Method for Convection-Diffusion-Reaction Problems
}

\author{
Yintzer Shih $\cdot$ R. Bruce Kellogg • Peishan Tsai
}

Published online: 16 April 2010

(C) Springer Science+Business Media, LLC 2010

Erratum to: J Sci Comput (2010) 43: 239-260

DOI 10.1007/s10915-010-9354-5

On page 3 of the original publication, the equation (5) was missing a symbol. The correct equation is as follows:

$$
\beta \cdot \nabla u^{r}+\gamma u^{r}=f
$$

The online version of the original article can be found under doi:10.1007/s10915-010-9354-5.

Y. Shih $(\bowtie) \cdot$ P. Tsai

Department of Applied Mathematics, National Chung Hsing University, 250 Kuo-Kuang Road, Taichung 40227, Taiwan

e-mail: yintzer_shih@email.nchu.edu.tw

R.B. Kellogg

Department of Mathematics, University of South Carolina, Columbia, SC 29208, USA 